\title{
Probiotics partially attenuate the severity of acute kidney injury through an immunomodulatory effect
}

\author{
Jihyun Yang ${ }^{1}$, Geun Eog Ji ${ }^{2,3}$, Myeong Soo Park ${ }^{2,3}$, Yeong-Je Seong ${ }^{3}$, Yoon Sook $\mathrm{Go}^{1}$, Hee Young Lee ${ }^{1}$, \\ Yina Fang ${ }^{1}$, Myung-Gyu $\mathrm{Kim}^{1}$, Se Won $\mathrm{Oh}^{1}$, Won Yong $\mathrm{Cho}^{1}$, Sang-Kyung $\mathrm{Jo}^{1}$ \\ ${ }^{1}$ Division of Nephrology, Department of Internal Medicine, Korea University College of Medicine, Seoul, Republic of Korea \\ ${ }^{2}$ Department of Food and Nutrition, Research Institute of Human Ecology, Seoul National University, Seoul, Republic of Korea \\ ${ }^{3}$ Research Center, BIFIDO Co. Ltd., Hongcheon, Republic of Korea
}

\begin{abstract}
Background: A healthy microbiome helps maintain the gut barrier and mucosal immune tolerance. Previously, we demonstrated that acute kidney injury (AKI) provoked dysbiosis, gut inflammation, and increased permeability. Here, we investigated the renoprotective effects of the probiotic Bifidobacterium bifidum BGN4 and the underlying mechanisms thereof.

Methods: C57BL/6 mice were subjected to bilateral renal ischemia-reperfusion injury (IRI) or sham operation. In the probiotic-treated group, BGN4 was administered by gavage once daily, starting 2 weeks before injury.

Results: Administration of BGN4 significantly increased gut microbiome diversity and prevented expansion of the Enterobacteriaceae and Bacteroidetes that were the hallmarks of AKI-induced dysbiosis. Further, BGN4 administration also significantly reduced other IRI-induced changes in the colon microenvironment, including effects on permeability, apoptosis of colon epithelial cells, and neutrophil and proinflammatory macrophage infiltration. Mononuclear cells co-cultured with BGN4 expressed significantly increased proportions of $\mathrm{CD}_{103^{+}} / \mathrm{CD}_{11} \mathrm{c}^{+}$and $\mathrm{CD} 4^{+} \mathrm{CD} 25^{+}$Treg cells, suggesting a direct immunomodulatory effect. BGN4 induced Treg expansion in colon, mesenteric lymph nodes (MNL), and kidney. BGN4 also reduced $\mathrm{CX}_{3} \mathrm{CR}_{1}{ }^{\text {intermediate }}$ Ly6C ${ }^{\text {high }}$ monocyte infiltration and interleukin (IL)-17A suppression in the small intestine, which may have attenuated AKI severity, kidney IL-6 messenger RNA expression, and AKI-induced liver injury.

Conclusion: Prior supplementation with BGN4 significantly attenuated the severity of IRI and secondary liver injury. This renoprotective effect was associated with increased Foxp3 and reduced IL-17A expression in the colon, MNL, and kidney, suggesting that BGN4-induced immunomodulation might contribute to its renoprotective effects. Probiotics may therefore be a promising strategy to reduce AKI severity and/or remote organ injury.
\end{abstract}

Keywords: Acute kidney injury, BGN4, Immunology, Microbiota, Probiotics

Received: December 21, 2020; Revised: June 3, 2021; Accepted: June 6, 2021

Editor: Kent Doi, The University of Tokyo, Tokyo, Japan

Correspondence: Sang-Kyung Jo

Division of Nephrology, Department of Internal Medicine, Korea University College of Medicine, Koreadae-ro 73, Sungbuk-gu, Seoul 02841, Republic of Korea. E-mail: sang-kyung@korea.ac.kr

ORCID: https://orcid.org/0000-0002-0496-0258

Copyright (@ 2021 by The Korean Society of Nephrology

(a) This is an Open Access article distributed under the terms of the Creative Commons Attribution Non-Commercial and No Derivatives License (http:// creativecommons.org/licenses/by-nc-nd/4.0/) which permits unrestricted non-commercial use, distribution of the material without any modifications, and reproduction in any medium, provided the original works properly cited. 


\section{Introduction}

Acute kidney injury (AKI) is a frequent complication in intensive care settings, especially among high-risk patients, including those with underlying comorbidities [1-3]. The occurrence of AKI is associated with an increased length of hospital stay, and greater mortality and economic burden. A recent meta-analysis found AKI to be a coronavirus disease 2019-associated complication, with an estimated incidence of $8.4 \% ; 3.6 \%$ of patients needed renal replacement therapy, and these patients had a 13-fold increased risk of mortality [4].

The human gut harbors more than 100 trillion microbial cells that maintain complete symbiosis with the host and play an important role in the development and shaping of the immune system $[5,6]$. The symbiotic relationship is altered in diverse pathological conditions and dysbiosis may be a key event in disease pathogenesis, given AKI provokes intense systemic inflammation with frequent distant organ injury $[7,8]$. Crosstalk between the kidney and gut in AKI has been postulated; however, unlike research into the pathogenesis of diabetes, obesity, or inflammatory bowel disease, there have been only a few studies that demonstrated links between the kidney and gut in AKI. Recently, we demonstrated a bidirectional relationship between the kidney and gut during AKI [9], such that AKI-induced dysbiosis was associated with increased gut permeability and bacterial translocation, intestinal inflammation, and reduced concentrations of short-chain fatty acids (SCFAs). Prior depletion of the gut microbiota by oral antibiotics resulted in less severe kidney injury and this renoprotective effect was associated with reduced intestinal Th17 cells and Th1 responses, along with expansion of Treg cells and M2 macrophages. We also demonstrated that intestinal microbiota act as an important modifier of post-AKI severity by showing that colonizing germ-free mice with microbiota harvested from post-AKI mice worsened kidney IRI, suggesting that the intestinal microbiota might be a novel therapeutic target $[9,10]$.

Probiotics are "live microorganisms" that have beneficial effects and have shown promising results in several chronic inflammatory conditions [8]. However, despite recent reports indicating possible renoprotective effects of probiotics or bacterial products, there have been few studies of the use of pre- or probiotics in AKI [11-15].

In this study, we investigated whether administration of probiotics had renoprotective effects in IRI-induced AKI. We chose Bifidobacterium bifidum BGN4 (BGN4) as the test probiotic. The genus Bifidobacterium is the predominant component of the intestinal microbiota and supports various functions including carbohydrate fermentation, vitamin synthesis, or immune modulation [16-18]. The effects of BGN4 on the severity of AKI and distant organ injury, as well as on the intestinal microenvironment, were investigated.

\section{Methods}

\section{Experimental animal model}

Six-week-old male C57BL/6 mice were obtained from Orient Bio Laboratory Animal Incorporation (Seoul, Korea). The mice were housed in a level 1 specific-pathogen-free facility, given ad libitum access to sterile food and water. The mice were randomized to the probiotic supplement + IRI or IRI-only groups. Probiotics were provided by BIFIDO Co. (Seoul, Korea), and BGN4 at a concentration of $2 \times 10^{9}$ was administered by gavage to each mouse in the probiotic supplement + IRI group once a day for 5 days a week (Fig. 1A). Two weeks later, the mice were subjected to IRI, for which the mice were administered intraperitoneal anesthesia using $50 \mathrm{mg} /$ $\mathrm{kg}$ ketamine and $0.04 \mathrm{~mL} / \mathrm{g}$ xylazine and then subjected to bilateral renal pedicle clamping for 25.5 minutes through a flank incision. A sham operation was also performed in a group of mice that underwent anesthesia and flank incision. All mice were placed on a heating pad and monitored until they woke up.

This study was approved by the Institutional Review Board of the Korea University College of Medicine Laboratory Animal Research Center. The study protocol was approved by the Animal Care Committee of Korea University (No. KOREA-2016-0260), and all animal experiments were performed in accordance with the National Institutes of Health (NIH) Guide for the Care and Use of Laboratory Animals and the NIH publication "Principles of Laboratory Animal Care."

\section{Serum chemistry and histological analyses}

Plasma creatinine, aspartate aminotransferase (AST), alanine aminotransferase (ALT), and lactate dehydrogenase (LDH) concentrations were measured using a Beckman 

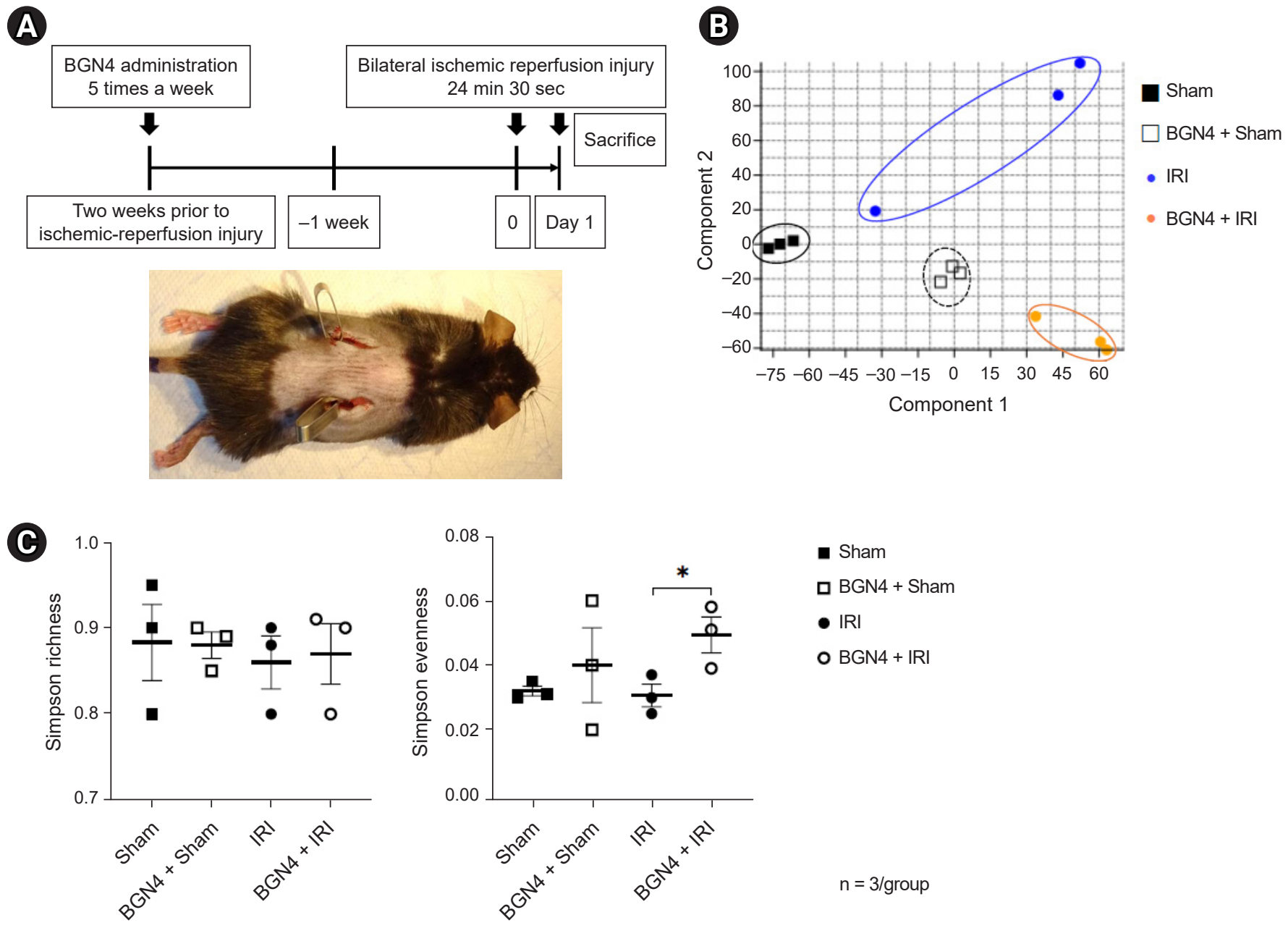

Figure 1. Microbiome and colon environmental alteration in acute kidney injury with BGN4 supplementation. (A) Treatment and experiment schedule for the ischemia-reperfusion injury (IRI) procedure. (B) Quantitative analysis of the microbiome showing the principal component of analysis using number of operational taxonomic units in each group. (C) Microbiome diversity index analysis in terms of richness and evenness using Simpson's methods. (D) Quantitative analysis of the microbial communities at the genus level was performed using 16S RNA isolated from stool samples. Only the genera with frequencies of $>1 \%$ and with significant differences between the groups were included. (E) Western blot analyses of claudin-1, occludin, and $\beta$-actin expression in the colon. (F) Western blot band intensities of claudin-1 and occludin in the colon normalized to those of $\beta$-actin. (G) Representative images of colon apoptosis using deoxynucleotidyl transferase dUTP nick end labeling (TUNEL) staining of the colon 1 day after IRI with semiquantitative comparison of the colon apoptosis-positive cells in each group. The number of positive cells per high-power field $(\mathrm{HPF})$ was compared $(\times 200)$. $(\mathrm{H})$ Representative images of Ly6G and F4/80 inflammatory cell infiltration into the colon $(\times 100)$. Semiquantitative comparison of each group according to the number of positive cells. (I) Intestinal permeability was measured by detecting the activity of fluorescein-isothiocyanate (FITC) 4 hours after the oral administration of FITC-dextran 1 day after IRI. $n=3-5$ per group. * $p<0.05$ compared with the sham vs. IRI. ${ }^{\#}<0.05$ compared with the IRI vs. BGN4 + IRI. (Continued to the next page)

AU 5821 chemical analyzer (Beckman Coulter, Brea, CA, USA) according to the manufacturer's instructions. The harvested kidney, liver, and colon tissues were fixed in $4 \%$ paraformaldehyde and embedded in paraffin. Kidney histology was assessed using standard light microscopy. Renal tubular injury was assessed in periodic-acid-Schiff-stained kidney tissues. Acute tubular necrosis was scored blindly by investigators who assessed the tubules affected by necrosis in five random areas throughout the tissue $(\times 100)$. Necrosis was assessed in a semiquantitative manner, with the differ- 


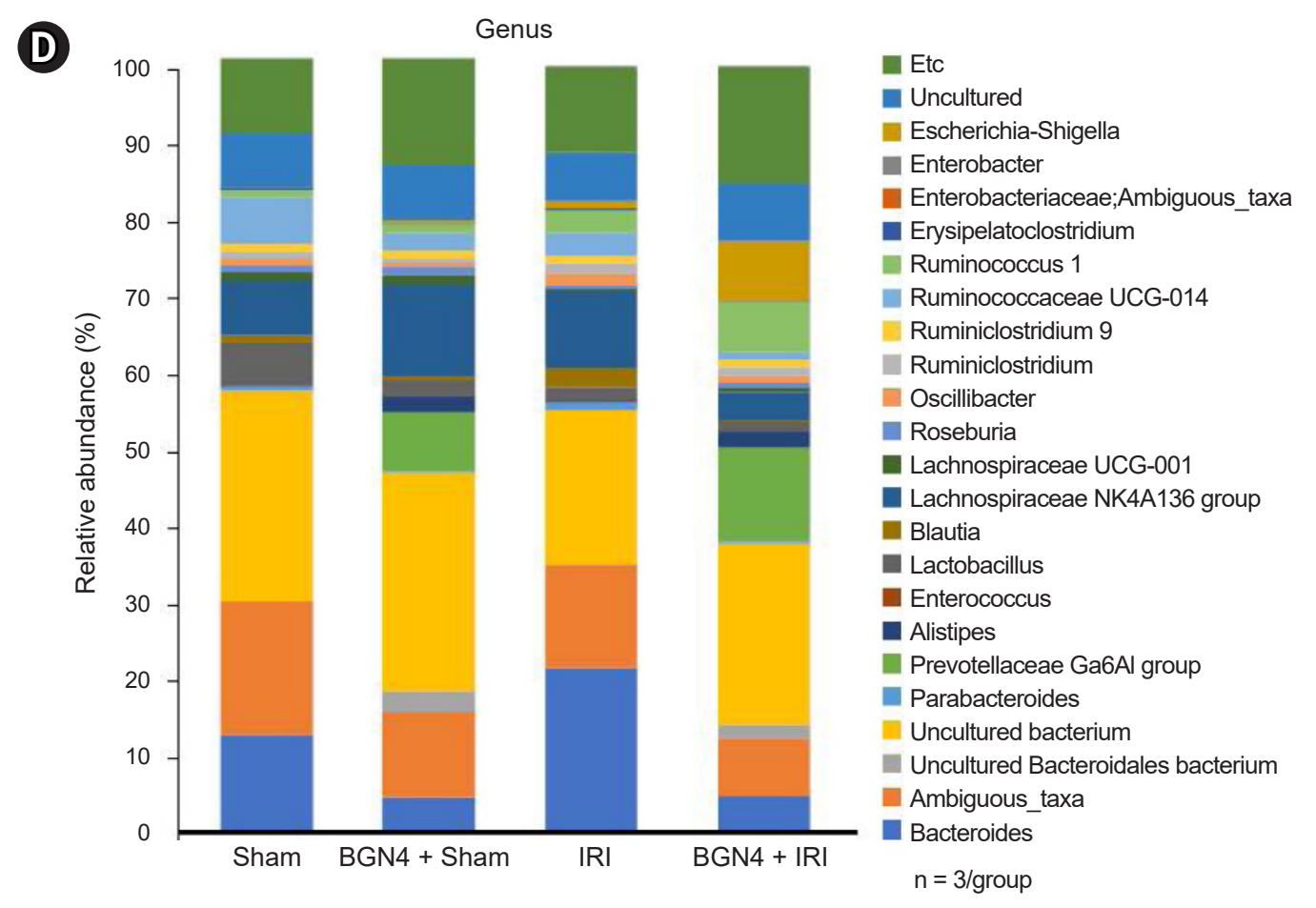

(E)

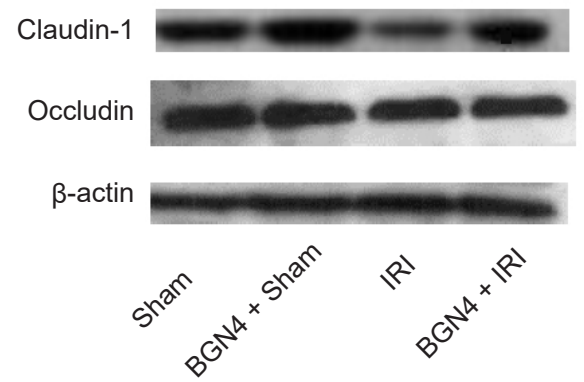

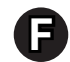

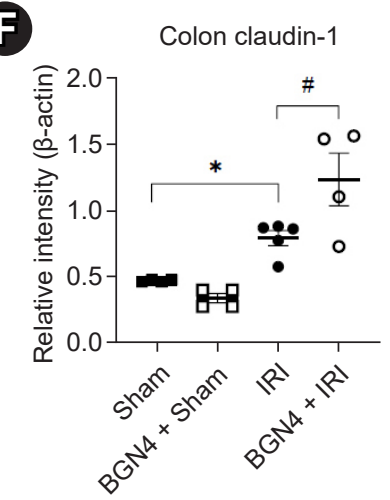

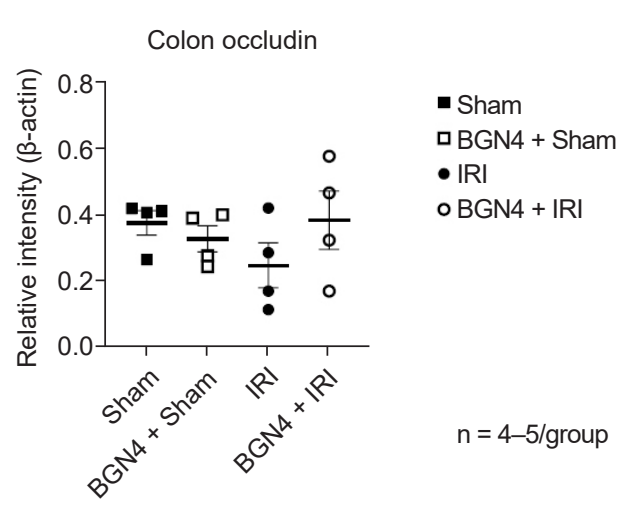

Figure 1. (Continued from the previous page)

ent grades assigned as follows: no visible necrosis (grade 0), $0 \%-25 \%$ (grade 1), $25 \%-50 \%$ (grade 2), $50 \%-75 \%$ (grade 3 ), or $75 \%-100 \%$ (grade 4 ) necrosis. To detect macrophage/ neutrophil infiltration, the kidney and colon tissues were stained with monoclonal antibodies against F4/80 (1:100; mch-497-GA; Bio-Rad Laboratories, Hercules, CA, USA) and Ly6G (1:200; 14-59-85; eBioscience, San Diego, CA, USA). To detect regulatory T cells (Tregs), immunohistochemical staining of the kidney and colon was performed using Foxp3 (1:1,000, ab215206; Abcam, Cambridge, UK). The mean numbers of positive cells per 10 high-power fields were compared. Colon epithelial cell apoptosis was assessed after terminal deoxynucleotidyl transferase dUTP nick end labeling (TUNEL) of epithelial cells by counting TUNEL-positive cells in 8-10 high-power fields $(\times 200)$. Liver tissues obtained from each group were stained using hematoxylin and eosin (H\&E). Images were collected at 20x magnification using a Slide Scanner automated image capture system (Axio Scan Z1; Zeiss Korea, Seoul, Korea).

\section{Western blot analyses}

Proteins were extracted from whole-colon tissue samples using the bicinchoninic acid method, and the expression 

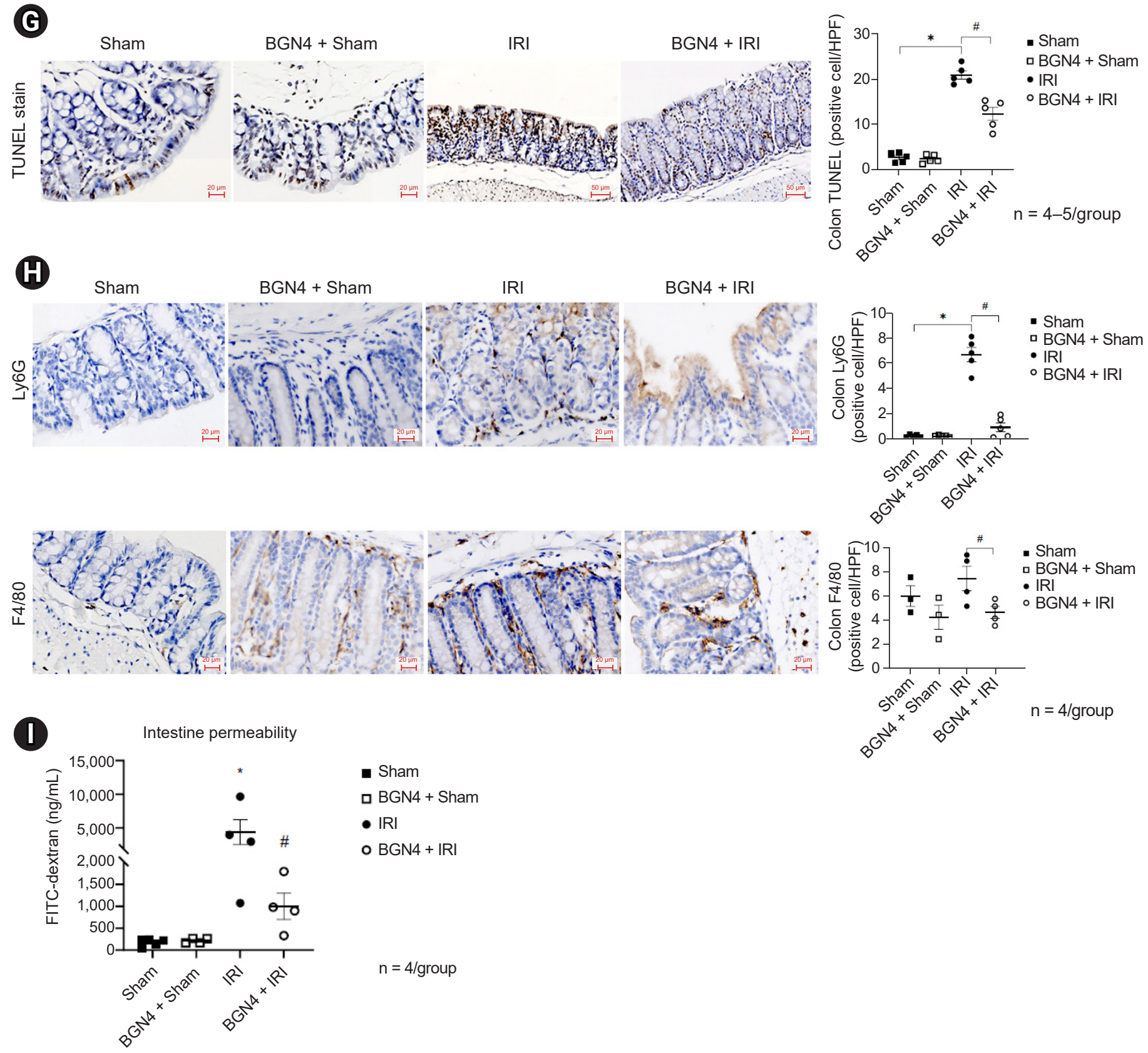

Figure 1. (Continued from the previous page)

of tight-junction proteins was examined using anti-mouse antibodies against claudin-1 (1:200; ab15098; Abcam) and occludin (1:200; ab168986; Abcam). The band intensities were measured using Image Studio Lite software (LI-COR; Biosciences, Lincoln, NE, USA). The target protein levels were normalized to those of $\beta$-actin.

\section{In vitro analyses}

Splenocytes $1.5 \times 10^{7}$ per well were incubated with BGN4 in $2 \%$ fetal bovine serum for 72 hours in a $5 \% \mathrm{CO}_{2}$ incubator

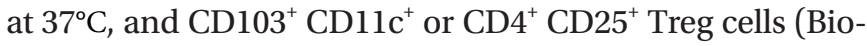
Legend, San Diego, CA, USA) were evaluated. 


\section{Intestinal permeability}

Fluorescein-isothiocyanate (FITC)-conjugated dextran (FITC-dextran; catalog number FD4; Sigma-Aldrich, St. Louis, MO, USA) dissolved in phosphate-buffered saline ( $100 \mathrm{mg} / \mathrm{mL})$ was administered by gavage $(44 \mathrm{mg} / 100 \mathrm{~g}$ ) after overnight water starvation, and the fluorescence activity of FITC in the blood was measured 4 hours later after first anesthetizing the mice. This method was performed as described previously [7].

\section{Flow cytometry}

Flow cytometric analyses of leukocytes in the mesenteric lymph nodes (MNL), spleen, kidney, and intestine were performed. The cells were stained with fluorochrome-labeled monoclonal antibodies (anti-CD4, anti-CD25, Fixable Viability Dye, anti-Foxp3, anti-CD45, anti-CD11c, anti-CD103, anti- $\mathrm{CX}_{3} \mathrm{CR}_{1}$, and anti-Ly6C; all eBioscience, BioLegend, or BD Biosciences [Franklin Lakes, NJ, US]) and analyzed using a four-color flow cytometer (FACSCanto II; BD Biosciences) and FlowJo software (Tree Star Inc., Ashland, CA, USA).

\section{Real-time reverse transcription-polymerase chain reaction}

For detection of Foxp3 messenger RNA (mRNA) expression, total RNA was purified using TRIzol extraction reagent (Thermo Fisher Scientific, Waltham, MA, USA) according to the manufacturer's protocol, and complementary DNA was synthesized using standard procedures. Real-time polymerase chain reaction was performed in an iCycler IQ Real-Time PCR Detection System Bio-Rad Laboratories (Hercules, California, USA) using the iQ SYBR Green Supermix (Bio-Rad Laboratories) for Foxp3. We used 18S ribosomal RNA (rRNA) as the reference gene (RT2 PCR Primer Set; Applied Biosystems, Foster City, CA, USA), and the fold difference was compared to that of the mice subjected to sham operation.

\section{Stool microbiome analyses}

More than two stool pellets per mouse were obtained and stored at $-70^{\circ} \mathrm{C}$. Microbiome analyses of pyrosequenced $16 \mathrm{~S}$ rRNA were completed using the Quantitative Insights
Into Microbial Ecology 2 (QIIME 2) database, which is a next-generation microbiome bioinformatics platform [19].

\section{Statistical analyses}

The data are presented as the mean \pm standard error of the mean. An unpaired t test and one-way analysis of variance (with Bonferroni post hoc test) were used for comparisons between two and three or more groups, respectively. A p-value of $<0.05$ was considered statistically significant. The data were analyzed using GraphPad Prism version 8.5 (GraphPad Software Inc., La Jolla, CA, USA).

\section{Results}

BGN4 modifies acute kidney injury-induced dysbiosis and enhances mucosal barrier function

The BGN4-supplemented group showed distinctive microbiome features in the principal coordinate analyses (PCoA) (Fig. 1B). One day after kidney IRI, the gut microbiome was characterized by relative expansion of Enterobacteriaceae and Bacteroidaceae families despite comparable diversity index (Fig. 1C). In contrast, BGN4 administration for 2 weeks prior to IRI increased microbiome evenness as measured using Simpson's diversity index, but did not significantly increase diversity (Fig. 1D), and also prevented the relative expansion of both marker species. These changes in the microbiome of the BGN4-supplemented group were associated with preservation of gut barrier integrity following IRI. Expression of the barrier-enhancing junctional protein claudin-1 was reduced after IRI and was partially restored in BGN4-supplemented mice, although the expression of junctional protein occluding- 1 did not change (Fig. 1E, F)

Apoptosis of colon epithelial cells was assessed semiquantitatively using TUNEL staining. The number of apoptotic cells increased significantly in IRI mice, but was reduced in BGN4-supplemented mice (Fig. 1G). In addition, the increase in the numbers of infiltrating Ly6G+ neutrophils and F4/80+ macrophages occurring after kidney IRI was significantly reduced in BGN4-pretreated mice (Fig. $1 \mathrm{H})$. And finally, the increase in gut permeability after IRI was partially reversed in BGN4-pretreated mice (Fig. 1I). 


\section{BGN4 attenuates severity of ischemia-reperfusion injury} and distant organ injury

Administration of the probiotic BGN4 for 2 weeks prior to IRI significantly attenuated kidney injury. Post-IRI tubular injury scores, as well as the number of neutrophils and macrophages and kidney interleukin (IL)-6 mRNA expression were significantly reduced in BGN4-pretreated mice (Fig. 2A, B). Plasma creatinine concentrations showed that BGN4 supplement mitigated IRI severity. We also found that BGN4 attenuated kidney-IRI-induced liver injury and reduced plasma LDH concentrations compared with those mice from the IRI-only group (Fig. 2C, D). The AKI-related distant organ injury was evaluated using liver histology $\mathrm{H} \& \mathrm{E}$ staining and plasma AST and ALT concentrations (Fig 2E). In BGN4-supplemented mice, no significant morphological alterations were noted in the liver, and the plasma AST and ALT concentrations indicated AKI-related liver damage was attenuated.

BGN-induced immunomodulatory effects might contribute to renoprotective effects

To investigate the mechanisms underlying the renoprotective effects of BGN4 pretreatment in experimental kidney IRI, we assessed the direct impact of BGN4 on immune cell function in splenocytes co-cultured with BGN4. This in vitro experiment showed that BGN4 facilitated expansion of $\mathrm{CD} 03^{+} \mathrm{CD} 1 \mathrm{c}^{+}$regulatory dendritic cells and of $\mathrm{CD} 4^{+}$ $\mathrm{CD}^{+} 5^{+}$regulatory $\mathrm{T}$ cells (Fig. $3 \mathrm{~A}$ ). Immunohistochemical examinations indicated increased kidney and colon Foxp3 staining in BGN4-pretreated mice compared to INI-only mice, and Foxp3 mRNA expression increased in both the colon and kidneys, suggesting that BGN4-induced Treg expansion may provide renoprotective effects in IRI (Fig. 3B, C). An increase in Foxp3+ Treg cells as measured using flow cytometry was evident in the kidney, MNL, and colon of BGN4-pretreated mice, consistent with the ameliorated $\mathrm{CX}_{3} \mathrm{CR}_{1}{ }^{\text {intermediate }} \mathrm{Ly}_{6} \mathrm{C}^{\text {high }}$ monocyte infiltration (Fig. 3D, E). Flowcytometry analysis showed CD4 ${ }^{+} \mathrm{IL}-17 \mathrm{~A}^{+} \mathrm{T} 17$ cell fraction was increased in IRI compared with sham, whereas the same fraction was decreased in the BGN4 + IRI. This result demonstrated BGN4 has a potential effect on the regulation of the Th 17 pathway (Fig. 3F).

\section{Discussion}

Accumulating evidence suggests that the gut microbiota and their metabolites regulate inflammation, oxidative stress, and fibrosis, and play pivotal roles in host physiology and pathology in kidney disease through activation of the gut-kidney axis. Here, we investigated the benefits of prior probiotic supplementation on AKI and the distant organ effects of AKI, and on the gut environment, in particular. Pretreatment of mice with BGN4 protected against AKI by reinforcing gut permeability and modulated the immune response to attenuate the severity of the AKI and distant liver injury.

Bifidobacterium is the dominant gut microorganism, accounting for more than $80 \%$ of the microbiota in the intestinal tract of healthy breast-fed infants; B. bifidum is the second most abundant species among Bifidobacterium. In contrast to breast-fed infants, formula-fed infants have a significantly lower prevalence of Bifidobacterium species. In addition, the abundance of $B$. bifidum decreases as an individual grows older. Various experiments have demonstrated that $B$. bifidum has a convincing beneficial effect. $\mathrm{Ku}$ et al. [17] demonstrated the immunomodulatory effect of BGN4 and its pharmaceutical application in a previous study. The BGN4 bacterium was first isolated from the feces of a breast-fed infant by Professor Geun Eog Ji and has since been used as a probiotic since $2000[17,18]$. In this study, BGN4 supplementation enriched the gut microbiome as shown by diversity evenness. Simpson's microbiome diversity index is an indicator that considers both the numbers of microbiome components and the abundance of the components. Along with the change in the microbiome, BGN4 supplementation induced changes in the intestinal environment toward a more renoprotective condition. Notably, BGN4 supplementation appeared to maintain colon barrier function by reinforcing tight-junction proteins, as well as reducing colon epithelial cell apoptosis and attenuating mucosal barrier disruption.

This study confirmed that the beneficial effects of probiotics are a function not only of prevention of pathogenic bacterial spread but also of direct strengthening of the intestinal barrier capacity. Preconditioning with BGN4 supplements can make the intense colon environment, protect the distant organ injury from AKI. In addition to directly strengthening mucosal barrier function, BGN4 produced 
(A)
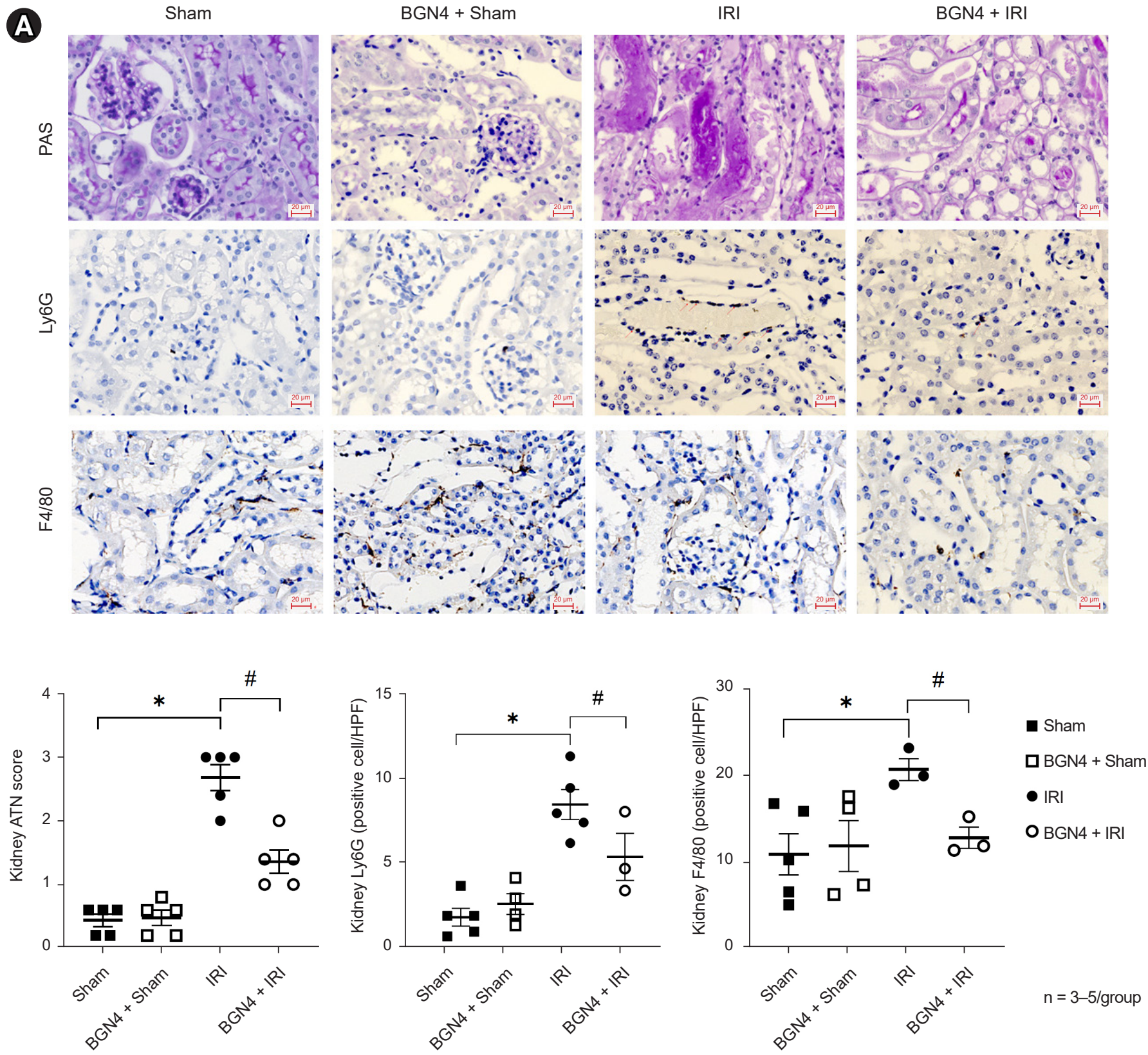

Figure 2. BGN4 attenuated ischemia-reperfusion injury (IRI) and distant organ injury. (A) Representative images of PAS-stained kidney tissue sections $(\times 100$ ), indicating the Ly6G- and F4/80-positive cells per high-power field (HPF). Tubular injury score, numbers of Ly6G and F4/80 positive cells of each group were compared. (B) The relative fold differences of kidney interleukin (IL)-6 messenger RNA expression were compared. (C) Serum creatinine concentrations in each group were compared. (D) Serum lactate dehydrogenase concentrations of each group were compared. (E) Live histology for each group as assessed using H\&E staining $(\times 200)$. Serum aspartate aminotransferase (AST) and alanine aminotransferase (ALT) concentrations of each group were compared. $n=3-5$ per group. ${ }^{*} p<0.05$ compared with the sham vs. IRI. ${ }^{*} p<0.05$ compared with the IRI vs. BGN4 + IRI. (Continued to the next page) 

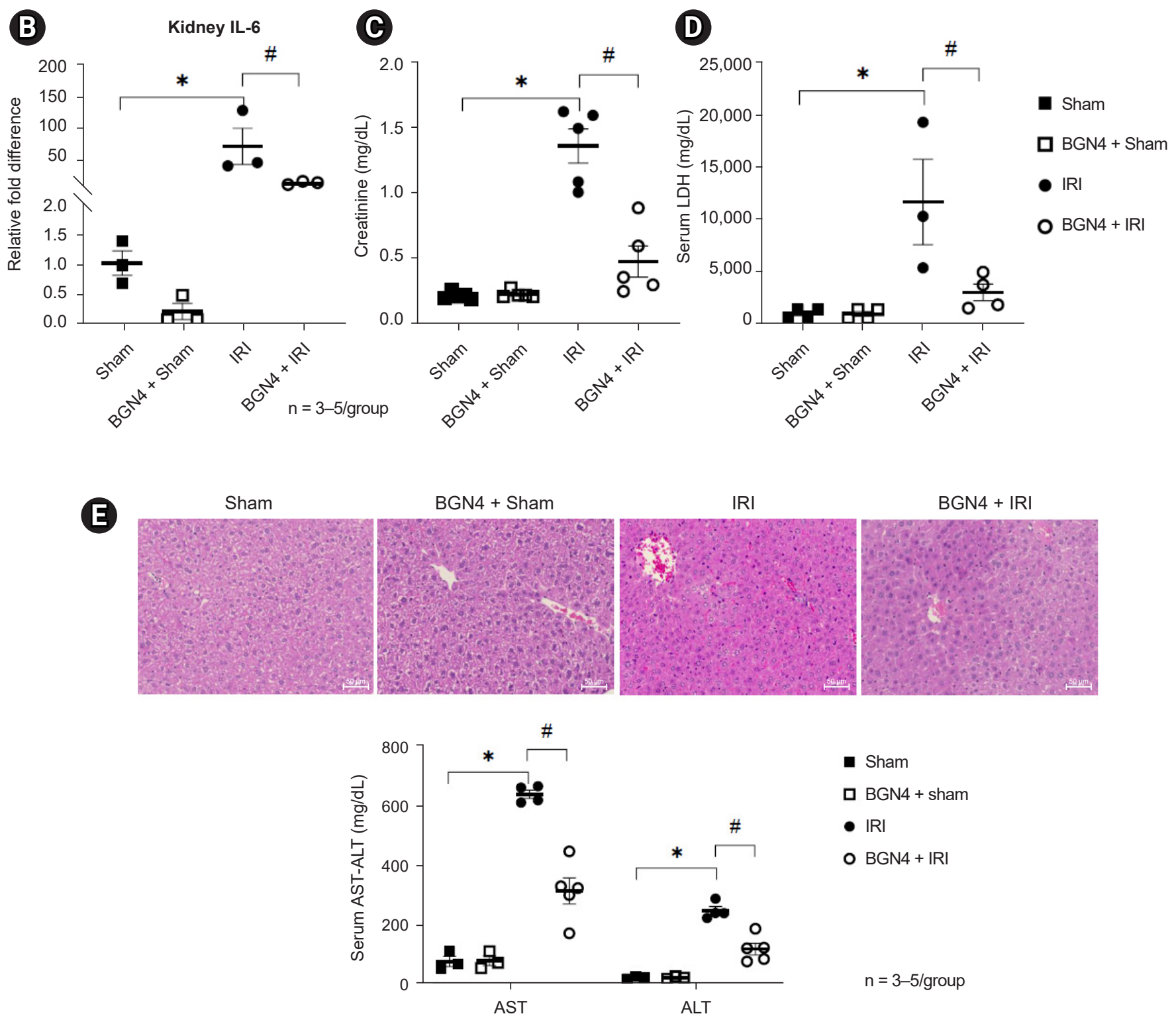

Figure 2. (Continued from the previous page)

immunomodulatory effects in the colon. The immunomodulatory effect of probiotics has been well studied in gastrointestinal disease like irritable bowel syndrome and allergic dermatitis. Previously, BGN4 was shown to induce macrophage differentiation [20], and to have anti-allergic effects by decreasing antigen-specific serum immunoglobulin $\mathrm{E}$ and IL-4 and increasing IL-12 concentrations [21]. We discovered another immune modulatory effect of BGN4, which was expansion of the colon regulatory $\mathrm{T}$ cell population. The protective role of regulatory $\mathrm{T}$ cell expansion has been examined in previous studies $[9,22,23]$. Administration of probiotics prior to IRI in the experimental setting may not be directly applicable to management of acute renal disease in a clinical setting. We did not conduct experiments assessing probiotic supplementation after IRI, and additional studies to assess the therapeutic potential of post-IRI BGN4 supplementation on IRI are needed.

Bifidobacteria are commensal bacteria that produce SCFAs. Supplementation with SCFAs attenuates kidney damage after IRI, colitis, and lung injury [12]. Supplementation with acetate-producing bacterial probiotics such as Bifidobacterium adolescentis and Bifidobacterium longum in AKI 

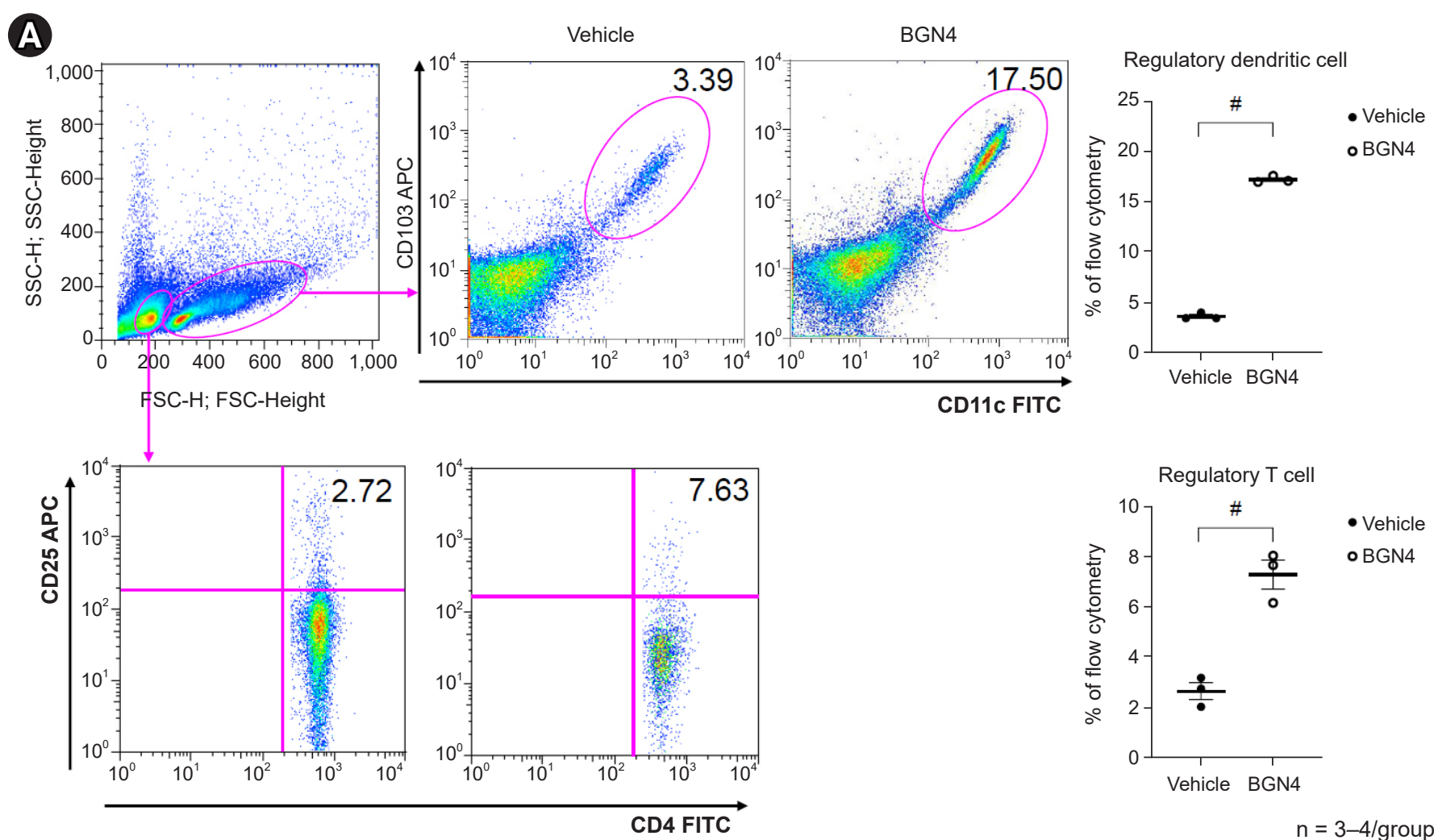

$n=3-4 / g r o u p$
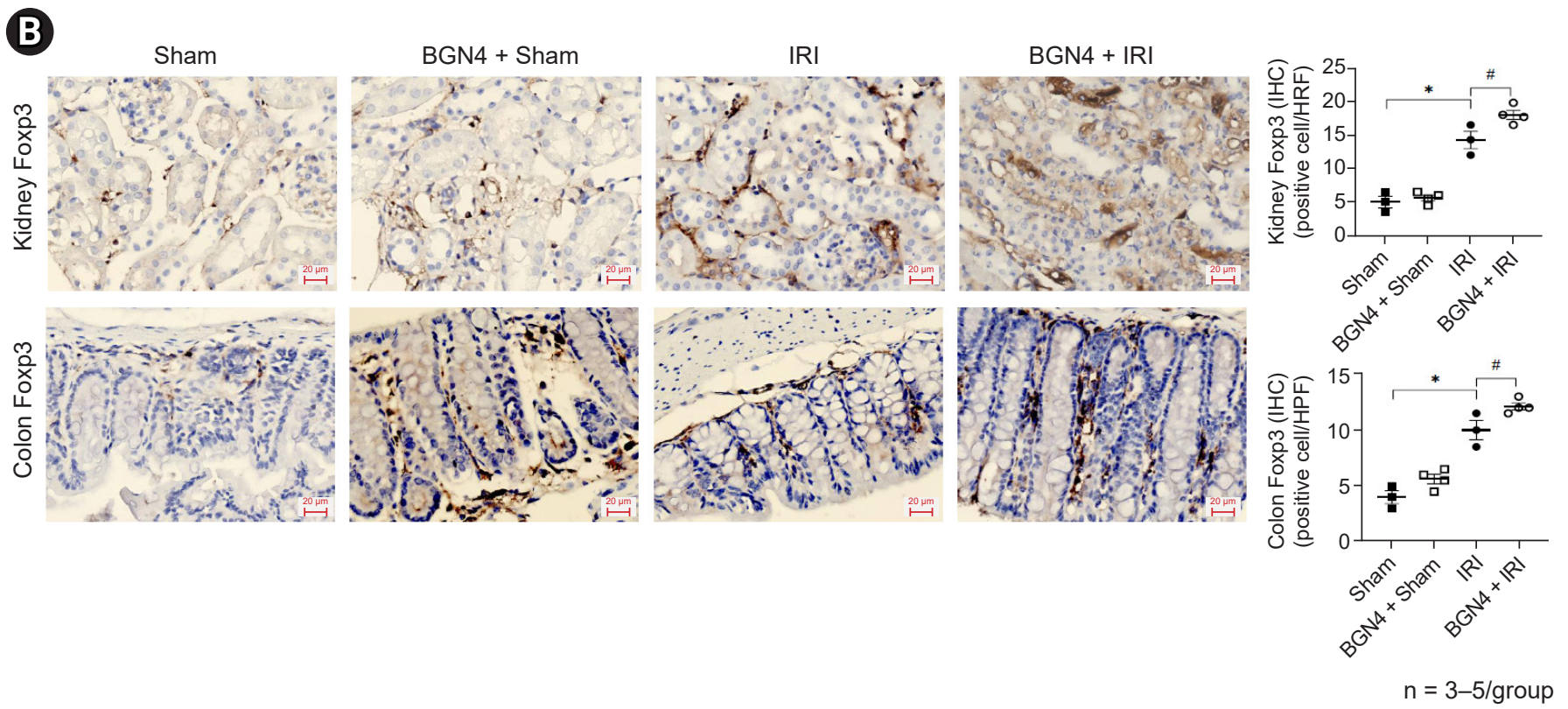

Figure 3. BGN4 modulates immune response in ischemia-reperfusion injury (IRI). (A) Flow cytometry of splenocyte cultured with BGN4 for 72 hours. Comparison of percent CD11c+CD103+ regulatory dendritic cells and CD $4^{+} \mathrm{CD} 25^{+}$regulatory $\mathrm{T}$ cells. (B) Representative images of kidney and colon Fopx3 staining $(\times 100)$, positive cells per high-power fields (HPF). Positive cells of each group were compared and shown a semiquantitative graph. (C) Relative fold difference of kidney and colon Foxp3 messenger RNA expression. (D) Flow cytometry of colon, mesenteric lymph node (MNL), and kidney of Foxp $3^{+} \mathrm{CD} 4^{+}$regulatory $\mathrm{T}$ cells in each group were compared. (E) Flow cytometry of colon $\mathrm{CX}_{3} \mathrm{CR}_{1}^{\text {intermediate }}$ Ly6 $\mathrm{C}^{\text {high }}$ monocyte. (F) Flow cytometry of small intestine interleukin (IL)-17A+ cells in each group were compared. $n=3-7$ per group. ${ }^{*} p<0.05$ compared with the sham vs. IRI. ${ }^{*} p<0.05$ compared with the IRI vs. BGN4 + IRI.

FITC, fluorescein-isothiocyanate; IHC, immunohistochemistry. (Continued to the next page) 



E Colon $\mathrm{CX}_{3} \mathrm{CR}_{1}^{\text {intermediate }} \mathrm{Ly} 6 \mathrm{C}^{\text {high }}$

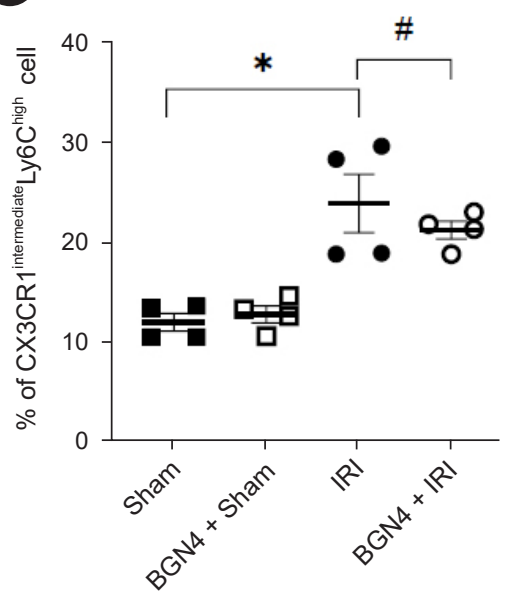

- Sham

ㅁ BGN4 + Sham

- IRI

- BGN4+ IRI

$\mathrm{n}=3-6 /$ group

Figure 3. (Continued from the previous page) 
(

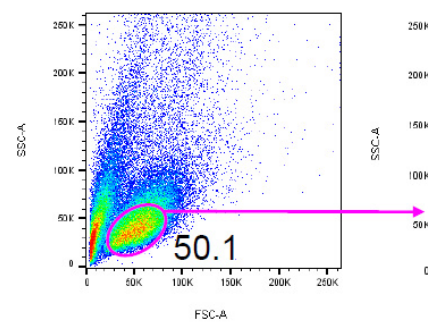

FSC.A
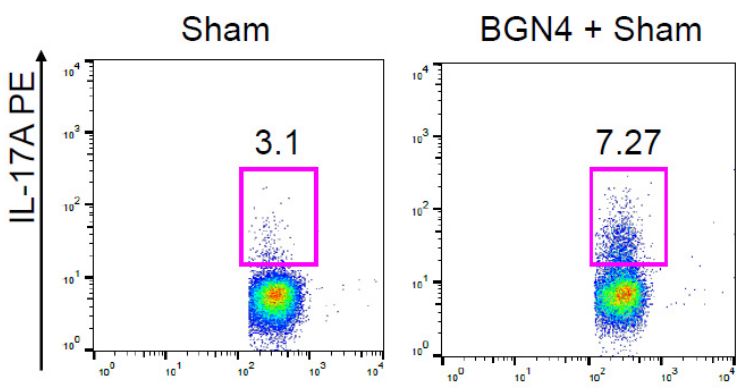

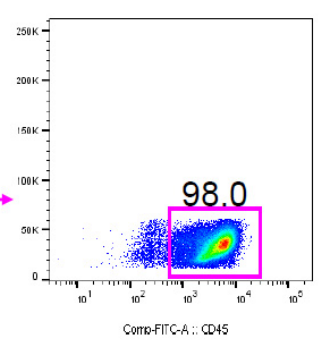

Comp-BV 510-A; FVD

COMnPFITS-A: : CD45

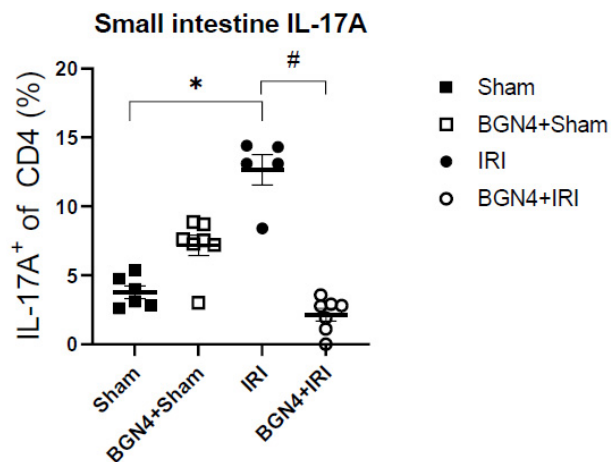

$\mid \mathrm{RI}$

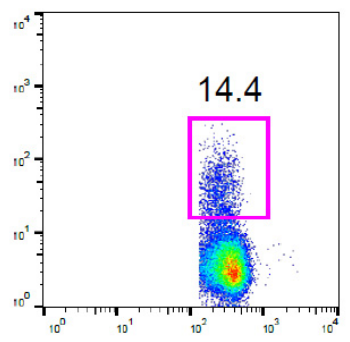

BGN4 + IRI

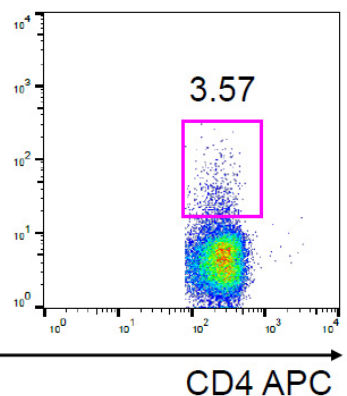

$n=5-7 / g r o u p$

Figure 3. (Continued from the previous page)

has also been shown to attenuate AKI-induced increases in serum creatinine [12]. Although we could not measure serum SCFAs (due to lack of facilities) in our study, we found that BGN4 pretreatment exerted a renoprotective effect by inhibiting inflammation and colon cell apoptosis. Intestinal permeability was reduced in mice supplemented with BGN4 prior to renal IRI, indicating that probiotics strengthen the colon mucosal barrier function. Post-IRI systemic inflammation and harmful effect were blocked as the colon barrier function was enhanced by probiotic supplementation. The serum LDH concentration is a marker of tissue damage and has been reported to be directly correlated with cardiovascular mortality and all-cause mortality in metabolic syndrome patients [24]. The increase in serum LDH concentrations occurring after AKI was also attenuated with BGN4 preconditioning. These findings demonstrated the beneficial effects of probiotics may in part be a function of reduced systemic inflammation. Nishida et al. [25] demonstrated that the renoprotective effect of recombinant thioredoxin-1 and albumin fusion protein in IRI also influenced AKI-associated distant organ injury, including injury to the lung and liver. Similarly, the renoprotective ef- fect of probiotic supplementation reduced AKI-related liver injury in our study. Although the liver histology as assessed using H\&E staining did not detect distinct histological differences between the IRI-only and BGN4-supplemented groups, the increase in plasma AST and ALT concentrations after IRI was attenuated in the BGN4 + IRI group. As probiotics function to strengthen the colon barrier and modulate the immune system, probiotics such as BGN4 may play a protective role in AKI-induced distant organ damage and in other diseases.

In conclusion, AKI-induced gut barrier disruption and colon inflammation may be among the mechanisms involved in systemic inflammation, kidney injury, and other remote organ injuries. Probiotic BGN4-mediated renoprotective effects may have occurred due to strengthening of the gut barrier and modulation of mucosal immune tolerance mechanisms. Probiotics may be a promising strategy to reduce the severity of AKI and/or remote organ injury.

\section{Conflicts of interest}

All authors have no conflicts of interest to declare. 


\section{Funding}

This study was supported by a Young Investigator Research Grant from the Korean Nephrology Research Foundation (KSN 2018), and a grant from the Korea University Anam Hospital, Seoul, Republic of Korea (K2014071).

\section{Authors' contributions}

Conceptualization: JY, GEJ, SKJ

Data curation, Formal analysis: MSP, YJS

Funding acquisition: JY

Investigation: YSG, HYL, YF

Methodology: JY, GEJ, SKJ

Project administration: MGK, SWO, WYC

Writing-original draft: JY, GEJ, SKJ

Writing-review \& editing: MGK, SWO, WYC

All authors read and approved the final manuscript.

\section{ORCID}

Jihyun Yang, https://orcid.org/0000-0002-8616-238X

Geun Eog Ji, https://orcid.org/0000-0002-6436-6824

Myeong Soo Park, https://orcid.org/0000-0003-4334-1457

Yeong-Je Seong, https://orcid.org/0000-0003-3048-036X

Yoon Sook Go, https://orcid.org/0000-0001-6165-4540

Hee Young Lee, https://orcid.org/0000-0001-9068-3068

Yina Fang, https://orcid.org/0000-0002-2375-3641

Myung-Gyu Kim, https://orcid.org/0000-0001-8807-2452

Se Won Oh, https://orcid.org/0000-0003-3795-9322

Won Yong Cho, https://orcid.org/0000-0003-1098-0252

Sang Kyung Jo, https://orcid.org/0000-0002-0496-0258

\section{References}

1. Case J, Khan S, Khalid R, Khan A. Epidemiology of acute kidney injury in the intensive care unit. Crit Care Res Pract 2013;2013:479730.

2. Fuhrman DY, Kane-Gill S, Goldstein SL, Priyanka P, Kellum JA. Acute kidney injury epidemiology, risk factors, and outcomes in critically ill patients 16-25 years of age treated in an adult intensive care unit. Ann Intensive Care 2018;8:26.

3. Lameire NH, Bagga A, Cruz D, et al. Acute kidney injury: an increasing global concern. Lancet 2013;382:170-179.

4. Hansrivijit P, Qian C, Boonpheng B, et al. Incidence of acute kidney injury and its association with mortality in patients with COVID-19: a meta-analysis. J Investig Med 2020;68:1261-1270.

5. Fung TC, Olson CA, Hsiao EY. Interactions between the microbiota, immune and nervous systems in health and disease. Nat Neurosci 2017;20:145-155.

6. Hooper LV, Littman DR, Macpherson AJ. Interactions between the microbiota and the immune system. Science 2012;336:12681273.

7. Doi K, Rabb H. Impact of acute kidney injury on distant organ function: recent findings and potential therapeutic targets. Kidney Int 2016;89:555-564.

8. Noel S, Martina-Lingua MN, Bandapalle S, et al. Intestinal microbiota-kidney cross talk in acute kidney injury and chronic kidney disease. Nephron Clin Pract 2014;127:139-143.

9. Yang J, Kim CJ, Go YS, et al. Intestinal microbiota control acute kidney injury severity by immune modulation. Kidney Int 2020;98:932-946.

10. Rabb H, Pluznick J, Noel S. The microbiome and acute kidney injury. Nephron 2018;140:120-123.

11. Patra A, Mandal A, Roy S, Mandal S, Mondal KC, Nandi DK. Protective effect of selected urease positive Lactobacillus strains on acetaminophen induced uremia in rats. Biomed Prev Nutr 2014;4:271-276.

12. Andrade-Oliveira V, Amano MT, Correa-Costa M, et al. Gut microbiota-derived D-serine protects against acute kidney injury. JCI Insight 2018;3:e97957.

14. Lee TH, Park D, Kim YJ, et al. Lactobacillus salivarius BP121 prevents cisplatin-induced acute kidney injury by inhibition of uremic toxins such as indoxyl sulfate and p-cresol sulfate via alleviating dysbiosis. Int J Mol Med 2020;45:1130-1140.

15. Iñiguez JS. Effect of probiotics and prebiotics in renal function in septic acute kidney injury patients [Internet]. 2019 [cited 2021 Jun 6]. Available from: https://clinicaltrials.gov/ct2/show/ NCT03877081.

16. Tojo R, Suárez A, Clemente MG, et al. Intestinal microbiota in health and disease: role of bifidobacteria in gut homeostasis. World J Gastroenterol 2014;20:15163-15176.

17. Ku S, Park MS, Ji GE, You HJ. Review on Bifidobacterium bifidum BGN4: functionality and nutraceutical applications as a probiotic microorganism. Int J Mol Sci 2016;17:1544.

18. Kim MJ, Ku S, Kim SY, et al. Safety evaluations of Bifidobacterium bifidum BGN4 and Bifidobacterium longum BORI. Int J Mol Sci 2018;19:1422.

19. Bolyen E, Rideout JR, Dillon MR, et al. Reproducible, interactive, scalable and extensible microbiome data science using QIIME 2. 
Nat Biotechnol 2019;37:852-857.

20. Kim NJ, Ji GE. Modulatory activity of Bifidobacterium sp. BGN4 cell fractions on immune cells. J Microbiol Biotechnol 2006;16:584-589.

21. Foroughi S, Foster B, Kim N, et al. Anti-IgE treatment of eosinophil-associated gastrointestinal disorders. J Allergy Clin Immunol 2007;120:594-601.

22. Kim MG, Jung Cho E, Won Lee J, et al. The heat-shock protein-70-induced renoprotective effect is partially mediated by CD4+ CD25+ Foxp3 + regulatory T cells in ischemia/reperfu- sion-induced acute kidney injury. Kidney Int 2014;85:62-71.

23. Alikhan MA, Huynh M, Kitching AR, Ooi JD. Regulatory T cells in renal disease. Clin Transl Immunology 2018;7:e1004.

24. Wu LW, Chen WL, Peng TC, et al. All-cause mortality risk in elderly individuals with disabilities: a retrospective observational study. BMJ Open 2016;6:e011164.

25. Nishida K, Watanabe H, Miyahisa M, et al. Systemic and sustained thioredoxin analogue prevents acute kidney injury and its-associated distant organ damage in renal ischemia reperfusion injury mice. Sci Rep 2020;10:20635. 